\title{
Lipossarcoma periorbital em paciente pediátrico: relato de caso
}

\section{Periorbital liposarcoma in pediatric patient: a case report}

\author{
Fernanda Marcio ${ }^{1}$, José Vital Filho ${ }^{1}$, Sylvia Regina Temer Cursino ${ }^{1}$, Patrícia Gomes Martins de Sousa ${ }^{1}$, Dino Martini Filho ${ }^{2}$
}

\section{RESUMO}

O objetivo desse estudo é descrever uma criança com lipossarcoma periorbital, caracterizando seus aspectos clínico-epidemiológicos e terapêuticos. Menina de 6 meses de idade com tumoração crescente há dois meses em região fronto-zigomática direita, a qual foi submetida à exérese e cujas análises anatomo patológica (AP) e imuno-histoquímica (IH) observaram achados típicos de lipoblastoma. Após isso, apresentou mais três recidivas tumorais com diagnósticos similares. Um ano depois da última cirurgia, houve nova recorrência, porém, dessa vez, o resultado dos exames análises anatomopatológica e imuno-histoquímica foi de lipossarcoma, sendo, então, encaminhada para complementar o tratamento com radio e quimioterapia, sem novas lesões até o momento. Devido a sua raridade, geralmente o lipossarcoma não entra no diagnóstico diferencial em pacientes com massas orbitais, porém, por ser localmente agressivo, torna-se vital a pronta identificação e tratamento de forma a oferecer melhores resultados terapêuticos e influência sobre a qualidade de vida do paciente.

Descritores: Lipossarcoma/radioterapia; Lipossarcoma/quimioterapia; Neoplasias orbitárias/cirurgia; Lipoblastoma; Marcadores biológicos de tumor; Humanos; Feminino; Lactente; Relato de caso

\begin{abstract}
The purpose of this study is report a child with periorbital liposarcoma describing the clinical, epidemiological and therapeutic aspects. Six-months-old female baby with increasing tumor in the right fronto-zigomatic region wich was submitted to excision and the patologic and immunohistochemistry analisys observed typical findings of lipoblastoma. After that, there were three tumors relapse with the same diagnosis. One year after the last surgery there was a recurrence of the tumor but at this time the diagnosis was lipossarcoma and the patient was referred for additional treatment wilth radiotherapy and chemotherapy no new injuries so far Due to its rarity, liposarcoma usually does not enter the differencial diagnosis in the patients with orbital masses, however because of its local aggressiveess, it's vital the early identification and treatment to provide better therapeutic results and quality of life.
\end{abstract}

Keywords: Liposarcoma/radiotherapy; Liposarcoma/drug therapy; Orbital neoplasms/surgery; Lipoblastoma; Tumormarkers, biological; Humans; Female; Infant; Case report

\section{INTRODUÇÃO}

Em relação a outros tipos de câncer, os sarcomas de tecidos moles são relativamente raros. O lipossarcoma constitui cerca de $9,8 \%$ a $18 \%$ dos sarcomas e é derivado de células primitivas que se diferenciam em tecido adiposo. A maioria dos casos de lipossarcoma orbital são primários e raramente metastáticos ${ }^{(1-5)}$.

O objetivo desse estudo é relatar uma criança com lipossarcoma periorbital, descrevendo seus aspectos clínico-epidemiológicos e terapêuticos.

\section{RELATO DO CASO}

Paciente de 6 meses de idade, do sexo feminino, foi encaminhada ao serviço com história de tumoração periorbital crescente há dois meses. Os antecedentes revelavam apenas polidactilia em mãos e pés.

À ectoscopia observava-se tumoração arroxeada, medindo 4,5 $\times 5 \mathrm{~cm}$, sem sinais flogísticos, na região fronto-zigomática direita. À palpação o tumor tinha consistência firme e era aderido a planos profundos. Ao exame oftalmológico, a paciente seguia objetos, não reagia à oclusão e apresentava ortotropia ao teste de Hirschberg. Os exames de motilidade ocular extrínseca, das reações pupilares e fundoscopia não revelaram alterações em ambos os olhos (Figura 1A).
A tomografia computadorizada da órbita evidenciou lesão hipodensa, arredondada, bem delimitada, na região fronto-zigomática direita, sem erosão óssea. A paciente foi submetida à exérese da lesão (Figuras 1B e 1C) e o exame anatomopatológico (AP) mostrou neoplasia coincidente com margens cirúrgicas e a análise imuno-histoquímica $(\mathrm{IH})$ demonstrou achados típicos de lipoblastoma, como proteína S100 focalmente positiva, vimentina positivo, além de desmina, AE1/AE3, CDE 34 negativos.

Após cinco meses, houve recidiva da lesão e feita nova ressecção. Nesse caso, o AP evidenciou aspecto agressivo com polimorfismo, presença de mitoses, os quais não existiam na lesão inicial e, adicionalmente, não se observava arquitetura lobulada que era presente anteriormente, e a $\mathrm{IH}$ apresentava vimentina difusamente positivo, S100 focalmente positivo, desmina e CD99 negativos, o que não contribuiu para concluir quanto à linha de diferenciação da proliferação. Diante da alteração desses aspectos, o caso foi enviado para Royal National Orthopedic Hospital em Londres (serviço de patologia especializado em lesões mesenquimais), onde foi realizado o teste de translocação FUS-CHOP, o qual foi negativo, corroborando com diagnóstico de lipoblastoma. Como desde a primeira cirurgia, as margens cirúrgicas eram comprometidas, houve recorrência tumoral mais duas vezes sempre com o mesmo diagnóstico. 

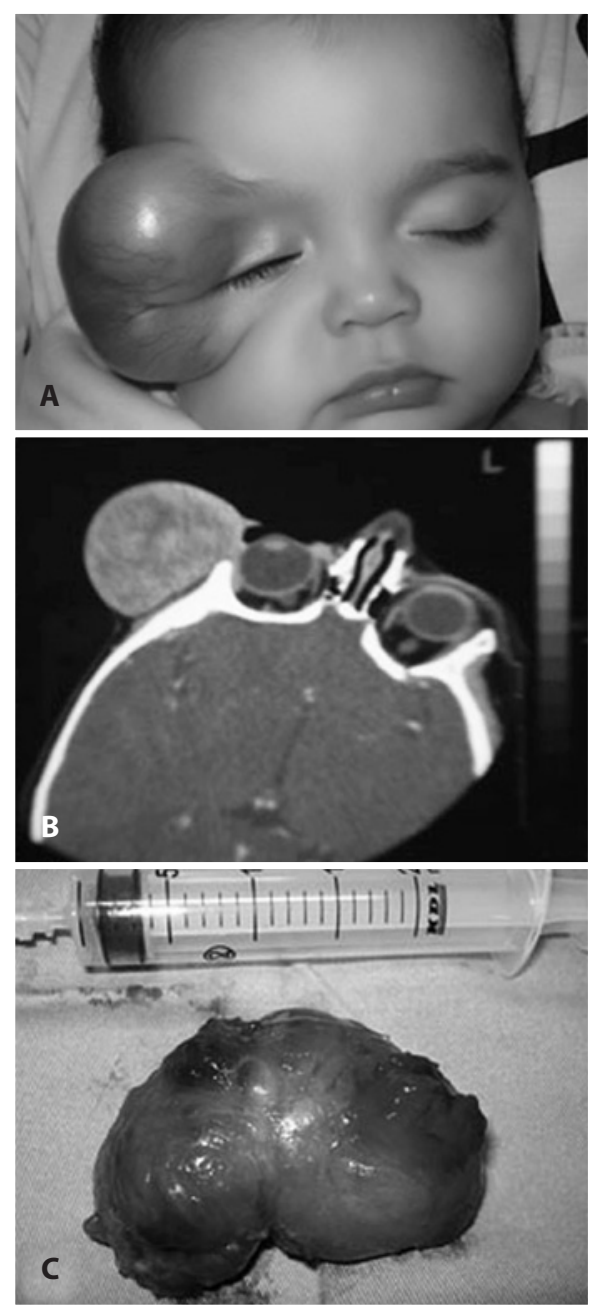

Figura 1. A) Tumoração arroxeada, de consistência firme e aderida, medindo $4,5 \times 5 \mathrm{~cm}$ de diâmetro, sem sinais flogísticos, em região fronto-zigomática direita. B) TC órbita: lesão hipodensa, arredondada, bem delimitada, localizada em região fronto-zigomática direita, sem erosão óssea. C) Exérese do tumor.
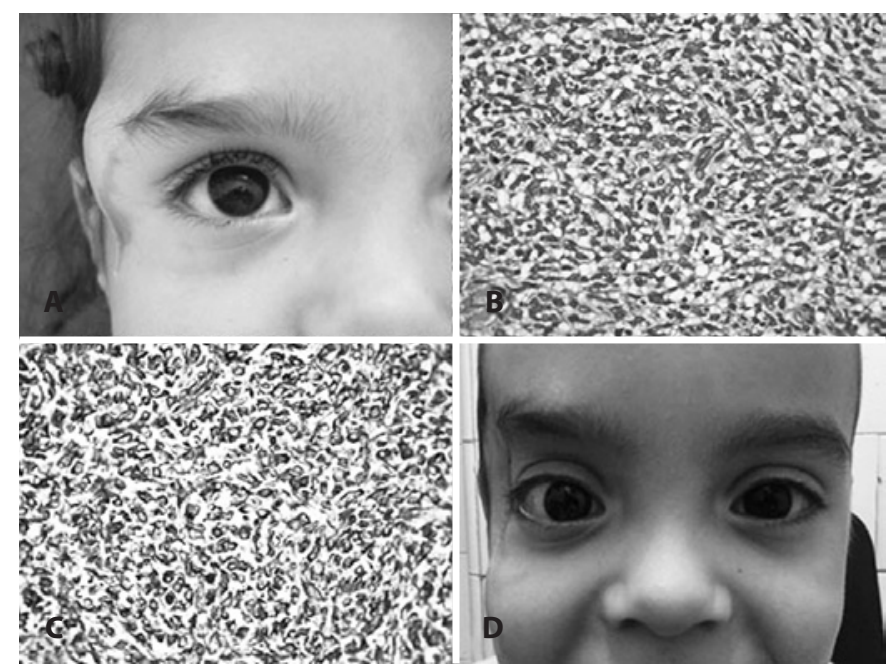

Figura 2. A) Segunda recidiva tumoral. B) HE: fenótipos epitelioide e fusocelular, focos de necrose alto índice proliferativo e áreas mixoides. C) Imuno-histoquímica: Vimentina positivo. D) Pós-operatório tardio da quinta cirurgia, sem indícios de recidiva tumoral até o momento.
Um ano após, com novo aparecimento da lesão, foi necessária outra intervenção cirúrgica (Figura 2A), sendo, desta vez, diagnosticado lipossarcoma mixoide devido à presença de focos de necrose, alto índice de proliferação, áreas mixoides, proteína S100 negativa e fenótipos epitelioide e fusocelular (Figura 2B e 2C), diferente das características dos tumores prévios que tinham fenótipo adipocítico, baixo grau histológico e imunoexpressão focal pela proteína S100.

Com avaliação junto à Oncopediatria, foram instituídos tratamentos radioterápico e quimioterápico, além de acompanhamento periódico semestral com avaliações clínicas e exames de imagem, sem recorrência tumoral ao longo dos quatro anos seguintes (Figura 2D).

\section{DISCUSSÃO}

Lipossarcoma é o sarcoma de tecidos moles mais comum em adultos e em geral afeta o retroperitôneo, coxa e região inguinal, mas é raro na órbita ${ }^{(1,4,5)}$. Apesar de raros, os sarcomas são tumores que também podem acometer a região da face e devem ser considerados como diagnóstico diferencial em lesões orbitais e periorbitais. Acredita-se que se desenvolva a partir de células mesenquimais em vez de um lipoma preexistente ${ }^{(6)}$.

É mais frequente no sexo masculino do que no feminino e na faixa etária entre 30-60 anos com idade média de aparecimento aos 53 anos de idade, sendo extremamente incomuns na infância ${ }^{(4,7)}$. Produz um quadro clínico que se assemelha a outros tumores orbitais, tais como proptose, distopia, diplopia, compressão do nervo óptico, dor e alteração da visão caso o tumor esteja no ápice orbital| $\left.\right|^{(1,4)}$.

Pode ser classificado nos seguintes cinco subtipos: bem diferenciado, mixoide, células redondas, indiferenciado e pleomórfico ${ }^{(1-3,7)}$. A maioria dos casos são tipo mixoide (30-50\% dos casos) ou bem diferenciado (20-30\%) e ambos têm um curso indolente ${ }^{(1,7)}$. A recorrência é comum, principalmente nos tipos pleomórfico e células redondas, entretanto, metástases locais ou à distância são raras ${ }^{(1,4,5)}$.

Histologicamente, o mixoide é caracterizado por matrix mixoide com lipoblastos e uma rede capilar delicada. Já o tipo bem diferenciado mostra predominância de linfócitos maduros e variedade de células fusiformes com núcleo hipercromático e lipoblastos com multiplos vacúolos ${ }^{(4,7)}$. Na imuno-histoquímica, apresenta $\$ 100$ e vimentina positivos e actina, miogenina e desmina negativos ${ }^{(7)}$.

Um diagnóstico diferencial é o lipoblastoma que é um raro tumor benigno dos tecidos moles. Este ocorre preferencialmente na infância, sendo 90\% dos casos em menores de 3 anos de idade, e, como o lipossarcoma, é mais frequente no sexo masculino(4). Histologicamente, o lipoblastoma apresenta tecido adiposo lobulado com septos fibrosos e estroma mixoide e lipoblastos imaturos, bastante semelhante com o lipossarcoma mixoide ${ }^{(6)}$. Na criança, o lipossarcoma e o lipoblastoma podem mimetizar hemangioma, diferenciando-se por meio da biópsia( ${ }^{(8)}$.

O tratamento de escolha do lipossarcoma é a exérese completa evitando, assim, a recidiva, que é comum. Além disso, os tipos mixoide são radiossensíveis, podendo, assim, ser utilizado como tratamento adjuvante, mas os bem diferenciados são menos responsivos à radioterapia ${ }^{(4,7)}$.

O uso da quimioterapia em lipossarcoma orbital não é bem descrito porém esquema de doxorrubicina e dacarbazina mostrou-se efetivo para o tipo mixoide(5)

Apesar de mais frequente em adulto e sexo masculino, nosso caso era uma criança no primeiro ano de vida e do sexo feminino, com tumor que recidivou cinco vezes e cujos primeiros diagnósticos foram de lipoblastoma, sendo que somente na quinta recorrência observaram-se características de lipossarcoma. Além das cirurgias, foi submetida à radioterapia e quimioterapia com doxorrubicina e isofosfamida com desaparecimento da lesão até o momento.

O caso atual evidencia que, embora o lipossarcoma seja um tumor raro na órbita e na criança, deve ser incluído no diagnóstico diferencial das massas orbitais, e, por ser localmente agressivo, tor- 
na-se vital a pronta identificação e tratamento de forma a oferecer melhores resultados terapêuticos e influência sobre a qualidade de vida do paciente. O caso serve ainda para mostrar que o diagnóstico anátomo-patológico pode ser difícil, podendo simular um lipoblastoma mesmo após várias recidivas.

\section{REFERÊNCIAS}

1. Al-Qahtani AA, Al-Hussain H, Chaudhry I, El-Khamary S, Alkatan HM. Primary orbital liposarcoma: histopathologic report of two cases. Middle East Afr J Ophthalmol. 2011; 18(4):314-6.

2. MacNab A, Moseley I. Primary orbital liposarcoma: clinical and computed tomographic features. Br J Ophthalmol [Internet]. 1990[cited 2012 Jul 21];74(7):437-9. Available from: http://bjo.bmj.com/content/74/7/437.long
3. Naeser P, Moström U. Liposarcoma of the orbit: a clinicopathological case report. Br J Ophthalmol[Internet]. 1982[cited 2012 Mar 19];66(3):190-3. Available from: http:// bjo.bmj.com/content/66/3/190.long

4. Mridha AR, Sharma MC, Sarkar C, Suri V. Primary liposarcoma of the orbit: a report of two cases. Can J Ophthalmol. 2007;42(3):481-3.

5. Madge SN, Tumuluri K, Strianese D, Bonavolonta P, Wilcsek G, Dodd TJ, et al. Primary orbital liposarcoma. Ophthalmology. 2010;117(3): 606-14.

6. Anantharajan N, Ravindranathan N. Parotid lipoblastoma in a child: Rare presentation as huge infratemporal mass with cervical extension. Indian J Plast Surg. 2010;43(1): 84-7.

7. Torrado CS, Zaldibar NM, Velasco-Benito V, Fernández-Hermida R. Primary orbital liposarcoma. J Craniofac Surg. 2011:22(3):1139-41.

8. Cai YC, McMenamim ME, Rose G, Sandy CJ, Cree IA, Fletcher CD. Primary liposarcoma of the orbit: a clinicopathologic study of seven cases. Ann Diagn Pathol. 2001; 5(5):255-66. 